\title{
A Simulational Performance of 5G MIMO Systems applying UFMC( Universal Filtered Multicarrier) Study
}

\author{
Preesat Biswas $^{1}$, Dr. Shanti Rathore ${ }^{2}$, Dr. M.R.Khan ${ }^{3}$ \\ ${ }^{1}$ Electronics and Communication Engineering Dr.C.V.Raman University Bilaspur, India \\ ${ }^{2}$ Electronics and Communication Engineering Dr.C.V.Raman University Bilaspur, India \\ ${ }^{3}$ Electronics and Telecommunication Engineering Government Engineering College Jagdalpur, \\ India1 preesat.eipl@gmail.com, ${ }^{2}$ rathoreshanti@gmail.com, ${ }^{3}$ mrkhan@gecjdp.ac.in
}

Article History: Received: 11 January 2021; Accepted: 27 February 2021; Published online: 5 April 2021

\begin{abstract}
The advanced wireless communication system uses the new technology, preparing the limited radio spectrum and its low expensive resource. The Scenario of Wireless communication is needed for the flexible mechanism with available time and frequency resources. The OFDM system, which is a multicarrier process with a remote framework in Long Term Evolution (LTE), 4G, and 5G. Using of UFMC(Universal_Filtered_Multicarrier)system with OFDM system symmetrical recurrence division multiply with modulation system and the correlation between them. Using of UFMC system with QAM into the MIMO system the result of subchannel, the model channel correlation recreated in "A Simulational Performance of 5G MIMO Systems applying UFMC (Universal Filtered Multicarrier) Study” result shown in MATLAB.

Keywords-UFMC; OFDM; MIMO; QAM; ZF; MMSE; 5G
\end{abstract}

\section{Introduction}

In present demanding of high data rates with new technologies, it might be used in new requirements [1]. In the recent scenario, the expectation of $5 \mathrm{G}$ and its offer is a great achievement. In new generation technology a great challenging issue of the mobile network for telecommunications professionals. The new service provider ties up 3GPP(3rd Generation Partnership Project) [2], the requirements are: URLLS (Ultra-Reliable Low Latency Communications), eMBB (Enhanced Mobile Broadband), mMTC (massive Machine Type Communications)

The implement of the $5 \mathrm{G}$ service provider's main aim is the flexibility that can offer connectivity growth of economical in the rural-urban area as well as agribusiness due to connectivity that is difficult to bring new technologies in this sector.

On the other hand, the previous network generation those are covering these regions was the high price of the spectrum with the use of the licensed band, and it is impossible to separate those who are invested in sparsely inhabited regions. This happens $5 \mathrm{G}$ range proposes reducing the network cost and allocated unlicensed of TVWS (TV-White Spaces) in VHF (Very High Frequency) and UHF (High Frequency).

One of the problematic in the OFDM system is tight timing and synchronization requirement because it will be burdening the lack of cyclic prefix leading [3] the technique also adds the effectual spectral

utilization via the diminishing of the interference between the subcarrier. Correcting the OFDM system using UFMC and multiply with RRC filter with $\mathrm{K}$ number of antenna, which is full capacity the transmission bandwidth through the use of the Offset Quadrature Amplitude Modulation (OQAM) [4]. This research will aim to compare the multicarrier of OFDM UFMC when applied for $5 \mathrm{G}$ wireless systems.

\section{System model}

We presented in this section the data are pass through UFMC in a subband symbol with a QAM system which is passed through in serial to the parallel system then passes through the N-IFFT and passes through with parallel to series and multiply with MIMO System.

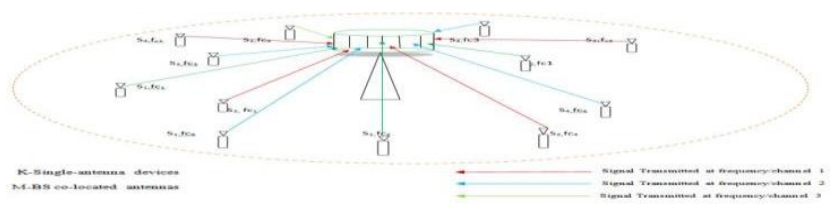




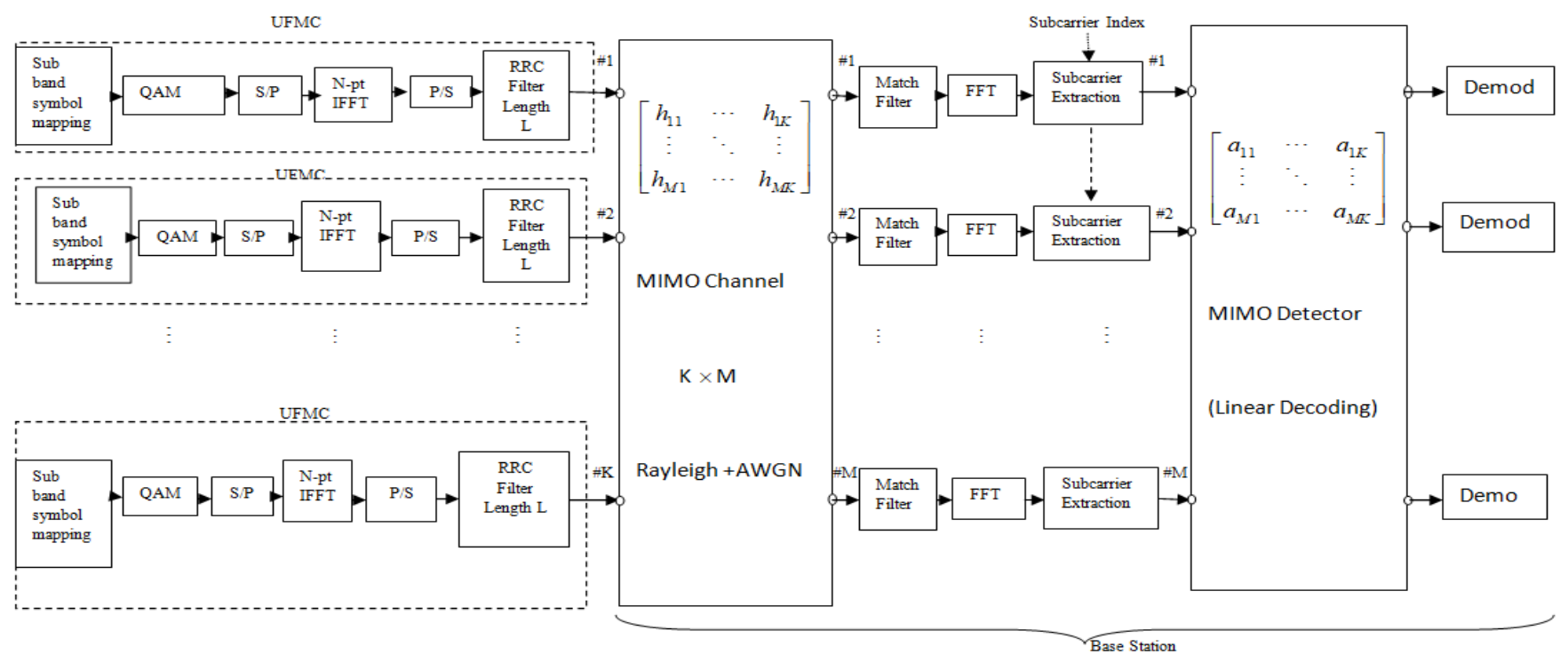

Fig. 2:- At the transmission side, $\mathrm{K}$ number of devices share the same time-frequency resource, while on the receiver side the BS is equipped with $M$ number of the antenna array.

The scenarios of MIMO system pass through the match which is multiplied with Rayleigh fading and AWGN. The match filter is connected to the FFT and subcarrier extraction system. The result of the incoming signal once again multiply with MIMO Detection. The frequency-domain get the result in window function and its inverse gives sync function express as

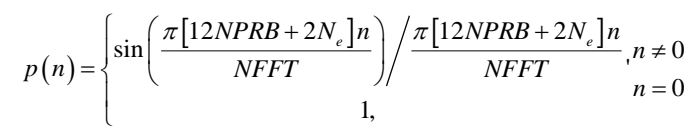

where NPRB, using of OFDM system is the number of physical blocks NFFT is the FFT length, and L is the $(L-1)$ (filter length) between $-(\mathrm{L}-1) / 2 \leq n \leq(\mathrm{L}-1) / 2$. The sub carriers at the left and right border those are excess bandwidth $\mathrm{Ne}$ in OFDM system symbols suffer less with attenuation.

However, it is impossible to implement a filter with such response, once it would require an infinite number of taps. Therefore, in order to obtain feasible filters, we truncate the sync's response by applying a window, $\mathrm{w}(\mathrm{n})$, which is defined next.

$$
w(n)=\left\{\frac{1}{2}\left[1+\cos \left(\frac{2 \pi n}{L-1}\right)\right]\right\}^{0.6}
$$

Finally, the normalized filter's coefficients are obtained as defined in Eq. (3),

$$
f(n)=\frac{p(n) \cdot w(n)}{\sum_{k} p(k) \cdot w(k)}
$$

The appropriate filter design should maintain ICI (Inter Carrier Interference) and ISI (Inter Symbol interference) interference at acceptable levels [8].

As we will see later, in the UFMC case, the filter length does not need to be smaller than the CP length. This is due to the fact that the kind of filter we employ has the majority of its energy concentrated in the main lobe of the Sinc square signal, which has length smaller than the CP length. Therefore, this allows filters with lengths 
longer than the CP one. Therefore, the energy leaking into the subsequent OFDM symbol's CP is very small and happens only for a short time [7].

\section{A. Detection Methods}

The scenario of 5G [2] and beyond networks. In the receiver side, the number $\mathrm{K}$ number of single antenna connected with BS devices is also equipped with M antennas and its mathematical expression Eq. (4),

$$
y=\mathbf{H} s+n
$$

Where $\mathrm{y}$ is the received signal vector in a $\mathrm{M} \times 1, \mathrm{H}$ is the channel matrix over in a $\mathrm{M} \times \mathrm{K}$, $\mathrm{s}$ is the transmitted signal vector in a $K \times 1$ and $n$ is the Gaussian noise vector in $M \times 1$. As we applying a MIMO system for the transmission of several signals over the same time-frequency resource and the estimated signal can be determined by using one of the techniques described below. 1) Maximum Ratio Combining (MRC): In this method, the signals from each antenna are summed, and the signal branch is weighted by a factor proportional to its power level [13]. In this way, the branches with stronger signal are amplified, while the ones with lower signal are attenuated. A BS employing

\subsection{Maximum Ratio Combining (MRC):}

MRC detection aims at maximizing the received signal-to noise ratio (SNR) of each device, neglecting the effects of multi-user interference (i:e:, cross-talk). On one hand it has low implementation complexity, however, on the other hand, it presents poor performance in interference-limited scenarios, once it ignores multi-user interference. The MRC detection is defined by the product between the Hermitian conjugate of the channel response, $\mathrm{H}$, and the received signal vector [14], according to Eq. (5).

1.2 Zero Forcing $(Z F)$ :

$$
\hat{s}=\mathbf{H}^{H} y
$$

The $\mathrm{ZF}$ detection technique is a suboptimal linear detection algorithm used in communications systems, which focuses on recovering the transmitted signal, by mitigating the interference among devices (i:e:, multiuser interference). The detection is achieved by applying the pseudo-inverse of the channel to the received signal, $y$ [12]. The ZF solution is found through Eq. (6),

$$
\hat{s}=\left(\mathbf{H}^{H} \mathbf{H}\right)^{-1} \mathbf{H}^{H} y
$$

ZF detection minimizes the interference between devices, but fails to tackle the effects of noise. Due to that fact, it performs well in interference limited scenarios and poorly in noise-limited scenarios. When compared to the MRC detector, it presents higher complexity, as it involves the computation of the pseudo-inverse of the channel matrices.

\subsection{Linear Minimum Mean Squared Error (LMMSE):}

LMMSE detection minimizes the Mean Squared Error (MSE) between the estimate A Hy and the transmitted signal s, where A is the MMSE detection matrix. The LMMSE detector mitigates device interference while also taking the noise effect into account. Therefore, LMMSE detection maximizes the received SINR (signal to interference plus noise ratio). Therefore, when compared to other sub-optimal detectors such as ZF, and MRC, MMSE is the one with the best detection performance. The LMMSE detection is found with (7).

$$
\hat{s}=\left(\mathbf{H}^{H} \mathbf{H}+\frac{\sigma_{n}^{2}}{\sigma_{s}^{2}} \mathbf{I}\right)^{-1} \mathbf{H}^{H} y(7)
$$

\subsection{Maximum Likelihood (ML):}

ML is the known to be optimal detection technique [14]. ML tests all values of $s$ and chooses the one with the smallest Euclidean distance to the received signal y, according to Eq. (8).

$$
\hat{s}=\underset{s \in \varsigma^{k}}{\arg \min }\|y-\mathbf{H} s\|
$$


where $\varsigma$ is the finite alphabet of $s_{k}, k=1,2, \ldots K$. The disadvantage of ML detection is that it has to search over the space of $|\varsigma|^{k}$ vectors, where $|\varsigma|$ is the cardinality of the set $\varsigma$. Therefore, its complexity exponentially increases with the number of devices and modulation order.

\subsection{OFDM (Orthogonal Frequency Division Multiplexing)}

In OFDM system, the arrangement signal system is divided into subcarriers in a repetitively system with a multicarrier filter bank scheme, which is substituted by the synthesis filter bank (SFB). Where in OFDM multicarrier are isolated into parallel information streams utilizing the arrangement parallel converter.

At the point in IFFT (Inverse Fast Fourier Transform) creating a period succession of the streams with CP broadens the time arrangement.

\subsection{UFMC (Universal Filtered Multicarrier)}

In UFMC technique with the OFDM system with the addition of the SRRC (square root rice cosine filter) pulse shaping filters that guarantee the localized sub channel in the frequency and time domain [9]. The UFMC system possesses which is eliminating the need for $\mathrm{CP}$ and offering more effective. In the transmitter side using of UFMC system working as a parallel form by the serial to parallel converter and transferred to the analysis filter bank [11].

\subsection{Difference between the OFDM and the UFMC techniques}

The new technique UFMC are used in with the OFDM and modulate the similar property in multicarrier system that created using the filter bank scheme by the synthesis filter bank (SFB).

\section{Comparing MMSE, $Z F$}

Evaluating the several MIMO detectors with FFT( Fast Fourier transform), LS , MMSE (Minimum Mean Squared Error), and comparing the equalize Zero Forcing (ZF), equalize Minimum Mean Squared Error(MMSE).

This paper is divided as follows: section II presents the system model, presents a model for comparison of the performance of MIMO systems when UFMC and OFDM waveforms are used with several detectors, the results and discussion in section III, and the conclusions are presented in section IV.

\section{RESULTS AND DISCUSSION}

In modulation system, the sample signal $105 \mathrm{~Hz}$ using of UFMC generalization the OFDM filtered entire band and create individual subcarriers are filtered in FBMC (Filter Bank Multi-carrier) in groups of subcarrier as shown in figure (3) and (4). Allow the subcarrier grouped which reducing the length, modifying the complex orthogonality with the help of QAM Pre-Equalization and existing MIMO schemes as shown in figure (5) . On the other hand the full band ' $\mathrm{N}$ ' is divided into subcarriers and each subband has a fixed number of subcarriers. Each subband is computed an N-pt IFFT, inserting zeros for unallocated carriers. L is the length of each subband filter and its responsibility adding all of them [1].

In receiving the process of UFMC system which like OFDM with FFT-based. The typical scenarios of persubcarrier equalization are used for equalizing the joint effect of the channel and the subband filtering. An equalized of UFMC channel does not affect if noise is added in receiver signal and we achieve the desired SNR (Signal to Nose ration)
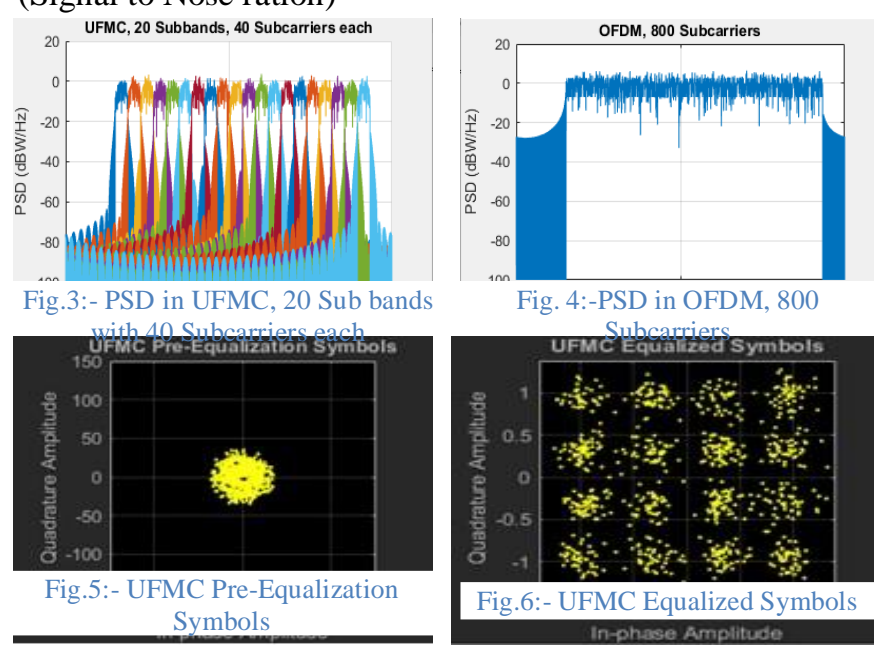


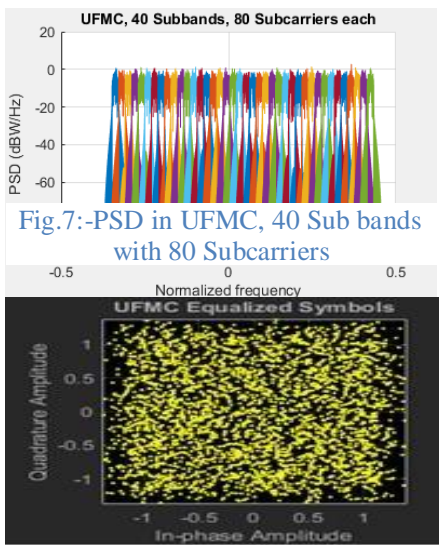

Fig.9:- UFMC Pre-Equalization Symbols

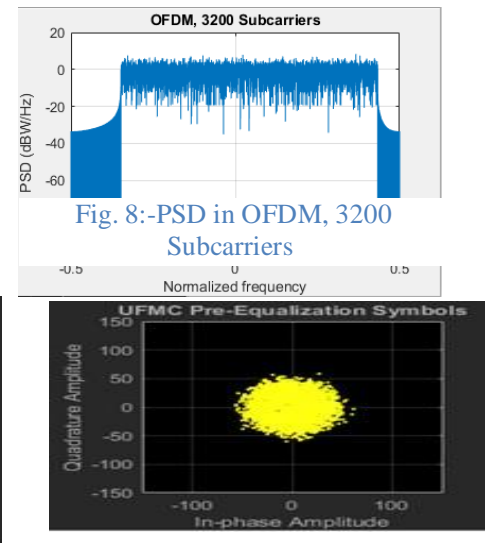

Fig.10:- UFMC Equalized Symbols

In the receiver side the data in 3-D numeric array or a 2-D numeric matrix, it has size-frequency window 27 with time window in modulation 64-QAM, where number of subcarrier is $\mathrm{N}$ and $\mathrm{M}$ is the number of OFDM symbol. The result of receive antennas is NR $\times$ Ants received as shown in fig. (6), \& (10). In the scenario of the physical channel index, the first two dimensions have been reduced to one dimension appropriate through the frequency and time locations of the resource elements of interest.
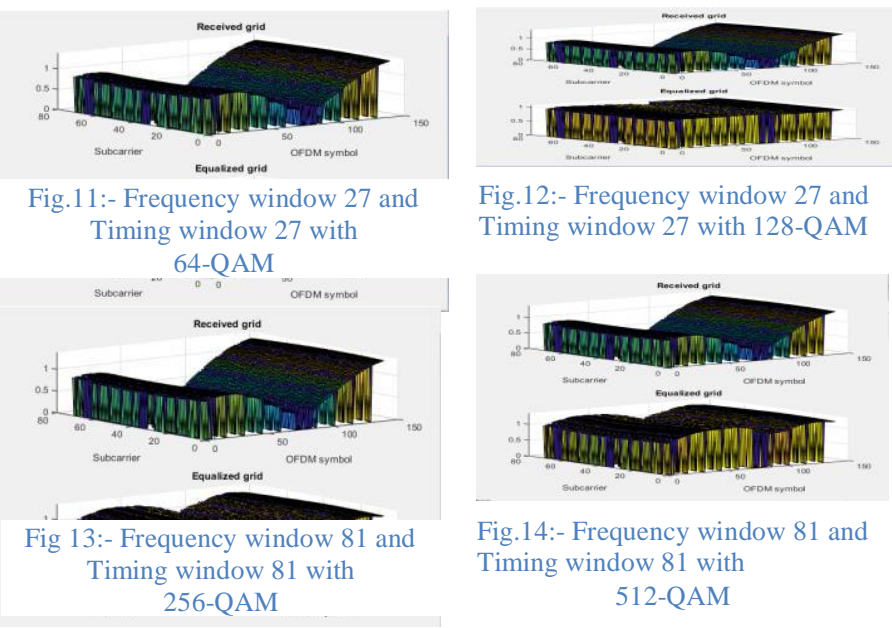

Fig.12:- Frequency window 27 and Timing window 27 with 128-QAM

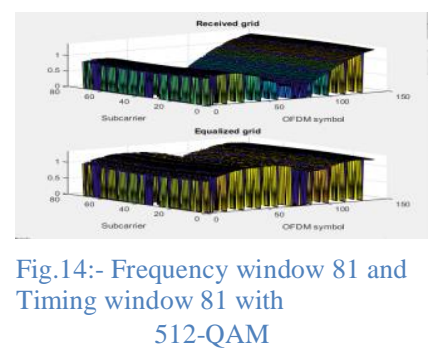

The variation of different QAM using in MMSE channel estimated with number of 80subcarrier in receiver side BER with SNR as shown in fig (15), (16) and (17). We compare the equalizer MMSE equalizer is better than the Zero force $(\mathrm{ZF})$ equalizer as shown in fig. (18)

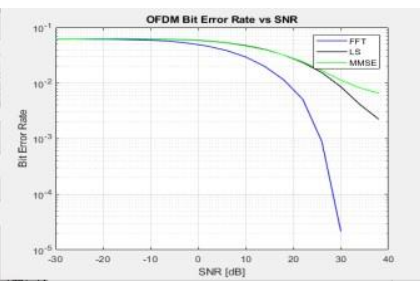

Fig. 15:- QAM-128 with $80=$ Subcarrier

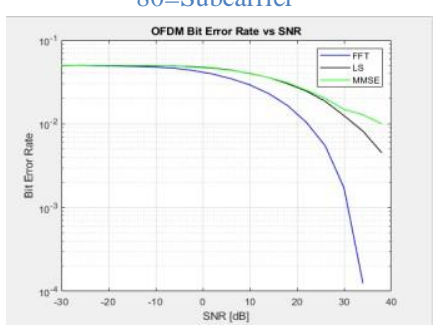

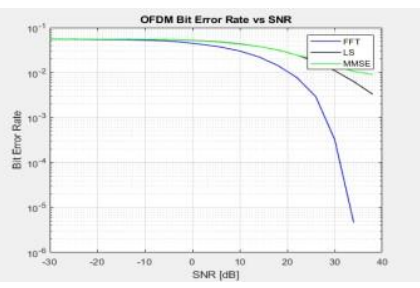

Fig.16:- QAM-512, 80=Subcarrier

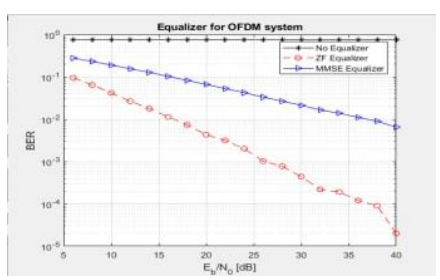


Fig. (19) and (20) shows that the (ACPR) adjacent channel power ratio base-band 5MHz using in QAM signal , having with FFT length of 256, compared the CCDF(Complementary Cumulative Distribution Function )using of 512-QAM and 1024-QAM with OFDM which is within the band limit .

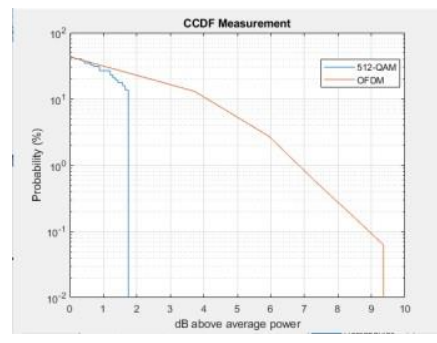

Fig. 19:- CCDF in QAM-512 with OFDM

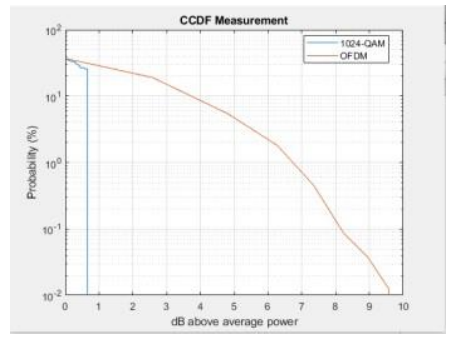

Fig.20:- CCDF in QAM-512 with OFDM

The table-1 shows that UFMC and OFDM in PAPR in receiver side, Pre- equalizer QAM and equalizer QAM are derive in BER in $5 \mathrm{MHz}$ frequency channel.

Using Power 15dB, ACPR (adjacent channel power ratio base-band) $5 \mathrm{MHz}$

Table-1

PERROTMANCE COMPARISION-1

\begin{tabular}{|c|c|c|c|}
\hline \multicolumn{4}{|c|}{ Adjacent Channel Power Ratio Base-Band with BER } \\
\hline & OFDM-PAPR & UFMC-PAPR & UFMC -BER \\
\hline 1 & 7.556 & 7.6466 & 0.005625 \\
\hline 2 & 9.9643 & 10.032 & 0.12453 \\
\hline
\end{tabular}

CCDF (complementary cumulative distribution function) with PAPR (Peak Power Output Port Using Power) using $15 \mathrm{~dB}$.

Table-2

PERROTMANCE COMPARISION-2

\begin{tabular}{|c|c|c|c|}
\hline \multicolumn{3}{|c|}{ Peak to Average Power Ratio between QAM and OFDM } \\
\hline & QAM & QAM PAPR in dB & OFDM PAPR in dB \\
\hline 1 & 64-QAM & 3.65 & 9.344 \\
\hline 2 & 512-QAM & 3.52 & 9.59 \\
\hline 3 & 1024-QAM & 4.50 & 9.84 \\
\hline
\end{tabular}

In the table -1 the result of PAPR both are uses OFDM and UFMC in power 5MHz but OFDM and UFMC are approximately same in case of 10 and its result more batter than the 7.5 in BER result. Where in the table- 2 increasing of OFDM-PAPR power $9.84 \mathrm{~dB}$ but QAM-1024 same cases power uses is only 4.50dB.The final result is using the QAM-1024 using in MIMO system with UFMC system is more reliable.

\section{CONCLUSIONS}

The scenario of in this paper, " A Simulational Performance of 5G MIMO Systems applying UFMC (Universal_Filtered_Multicarrier ) Study" the incoming signal subband system using of QAM pass through OFDM with UFMC modulation system, where we convert to serial to parallel system then pass through N-pt IFFT and once ageing convert to parallel to serial multiply with RRC filter with K number of antenna. UFMC system has subband with subcarrier system which is connected to MIMO system. The MIMO system match filter was analyzed and detect the ZF, MMSE, LS. The subcarrier system is extracted and those do not affect equalizer 
ZF, equalizer MMSE, and No equalizer system. At the end of the result using of low power PAPR of UFMC integrating with QAM system good performance for the future generation of wireless and mobile networks.

\section{REFERENCES}

1. Felipe A. P. de Figueiredo, R. Mennes, X. Jiao, W. Liu, and I. Moerman, A spectrum sharing framework for intelligent next generation wireless networks, IEEE Access, vol. 6, pp. 60704-60735, Nov. 2018.

2. 3GPP, Technical enhancements and improvements for rel-15, 3rd Generation Partnership Project (3GPP), Technical Specification (TS) 21.915, Jun 2015.

3. G. Alcoz, "Next Generation 5G OFDM-Based Modulations for Intensity Modulation-DirectDetection (IMDD) Optical Fronthauling," Technical University of Catalonia, Barcelona, Spain, 2017.

4. T. E. A. V. Agrell, E. and K. Zeger., Closest point search in lattices, IEEE Transactions on Information Theory, vol. 48, no. 8, pp. 2201-2214, Aug. 2002.

5. Saadaldeen Rashid Ahmed1, Ahmed S.Abdullah2 , Nayif Mohammed Hammash3,Journal of Physics: Conference Series 1530 (2020) 012092 IOP Publishing doi:10.1088/1742-6596/1530/1/012092

6. S. B. Weinstein, Introduction to the history of OFDM, IEEE Commun. Mag., vol. 47, no. 11, pp. 26-35, 2009.

7. Y. Li, J. H. Winters, and N. R. Sollenberger, MIMO-OFDM for wireless communications: Signal detection with enhanced channel estimation, IEEE Trans. Commun., vol. 50, no. 9, Sept. 2002.

8. Mansoor Shafi, Andreas F. Molisch, Peter J. Smith, Thomas Haustein, Peiying Zhu, Prasan De Silva, Fredrik Tufvesson, Anass Benjebbour, and Gerhard Wunder, 5G: A Tutorial Overview of Standards, Trials, Challenges, Deployment, and Practice, IEEE Journal on Selected Areas in Communications, vol. 35, no. 6, pp. 1201-1221, June 2017.

9. L. Zhang, A. Ijaz, P. Xiao, M. M. Molu, and R. Tafazolli, Filtered OFDM systems, algorithms and performance analysis for 5G and beyond, IEEE Trans. Commun., vol. 66, no. 3, pp. 1205-1218, Nov. 2017.

10. J. Abdoli, M. Jia, and J. Ma, Filtered OFDM: A new waveform for future wireless systems, in 2015 IEEE 16th International Workshop on Signal Processing Advances in Wireless Communications (SPAWC), 2015.

11. 3GPP, 3rd Generation Partnership Project; Technical Specification Group Radio Access Network; Evolved Universal Terrestrial Radio Access (EUTRA); Physical layer procedures (Release 8), 2008.

12. 3GPP, LTE; Feasibility study for Further Advancements for E-UTRA (LTE-Advanced) (Release 9), TR 36.912, 2009.

13. Klein, G. Kawas Kaleh, and P. Walter Baier, Zero forcing and minimum mean-square-error equalization for multiuser detection in code-division multiple-access channels, IEEE Trans. Veh. Technol., vol. 45, no. 2, pp. 276-287, May 1996.

14. K. Tiwari and D. Saini, SER improvisation of mimo-mrc system over weibull-gamma fading channel, in 2015 International Conference on Signal Processing and Communication (ICSC), pp. 70-73, March 2015.

15. F. Labaran, Spatial modulation: A comparison of maximum receiver ratio combining and maximum likelihood detectors, in $201411^{\text {th }}$ International Conference on Electronics, Computer and Computation (ICECCO), pp. 1-3, Sep. 2014.

16. U. Fincke, and M. Pohst, Methods for calculating vectors of short length in a lattice, including a complexity analysis, Mathematics Subject Classification, vol. 44, pp. 463-471, 041985.

17. P. Kansal and A. K. Shankhwar, "FBMC vs. OFDM Waveform Contenders for 5G WirelessCommunication System," Wireless Engineering and Technology, vol. 8, no. 4, pp. 59-70, 2017.

18. R. Nissel, S. Schwarz and M. Rupp, "Filter Bank Multicarrier Modulation Schemes for FutureMobile Communications," IEEE Journal on Selected Areas in Communications, vol. 35, no. 8, pp. 1768-1782, 2017.

19. M. Saad, A. C. Al Ghouwayel and H. Hijazi, "UFMC Transceiver Complexity Reduction," in 25thInternational Conference on Telecommunications, Saint-Malo. France, 2018.

20. J.-B. Dore, R. Gerzaguet, N. Cassia and D. Ktenas, "Waveform contenders for 5G: Description,analysis, and comparison," Physical Communication, vol. 24, pp. 46-61, 2017.

21. D. M. N and A. V. Singh, "An Efficient Orthogonal Frequency Division Multiplexing (OFDM)System and Performance Analysis of Digital Audio Broadcasting (DAB) System," International Journalof Computer Applications, vol. 148, no. 8, pp. 12-16, 2016.

22. Zaier and R. Bouallegue, "A Full Performance Analysis of Channel Estimation Methods for Time-Varying OFDM Systems," International Journal of Mobile Network Communications \& Telematics, vol. 1, no. 2, pp. 1-20, 2011.

23. R. Balla, A. Chadha and N. Satam, "Orthogonal Frequency Division Multiplexing and its Applications," International Journal of Science and Research, vol. 2, no. 1, pp. 325-328, 2013

24. W. Shahjehan, M. H. Zafar, I. Hussain, K. Ahmad, N. Iqbal, and F. Altaf, "Universal Filtered Multicarrier for 5G," International Journal of Engineering Works, vol. 4, no. 7, pp. 136-139, 2017. 
25. S. N. S, "Implementation and Study of Universal Filtered Multi-Carrier under Carrier Frequency Offset for 5 G," IPASJ International Journal of Electronics \& Communication, vol. 4, no. 4, pp. 1-5,2016.

26. Jafri, J. Majid, L. Zhang and M. A. Imran, "FPGA Implementation of UFMC Based Baseband Transmitter: Case Study for LTE 10MHz Channelization," Wireless Communications and Mobile Computing, no. 5, pp. $1-12,2018$. 